\title{
Study with surface electromyography, borg scale and rom upper trapezius, deltoid of the three portions (clavicular, acromial, spinal) and the latissimus dorsi muscle, before and after using a muscle activation technique (mat)
}

\author{
ESTHER BLASCO ${ }^{1}$, VICENTE LIZONDO ${ }^{1}$, LUIS BERBEL ${ }^{2}$, JULIO MARTIN ${ }^{1}$ \\ 1 Universidad Católica de Valencia, San Vicente Martir, Spain \\ 2 Neogym Center Valencia, Spain
}

\begin{abstract}
The pathologies located at shoulder can be caused by misuse or continued implementation of a sporting practice or physical activity. These pathologies are second position in the ranking of Traumatologics and Orthopedic Clinicals (Windt, Koes, De Jong \& Bouter, 1995). Shoulder's muscle injuries can occur when the arm is in a position that exceeds normal, this fact is repeated in sport, may be the repetition of the hand signal of the inductor problem (Kapandji, 2006). Given the importance of muscle function of the shoulder joint, along the existing boom of new techniques in the specific muscle activation has been carried out an experimental study using a " Test neuro proprioceptive response " with other scientific techniques (EMG, Borg Scale and ROM), which will provide us information about the activation of the upper trapezius, acromial, scapular, spine, latissimus dorsi. This study had 5 professional volunteered women specialists of Physical Activity, which to analyze them, their muscle activation. The results show that all the muscles analyzed shows significant improvement $(p<.05)$ in mean muscle activation recorded with electromyography in the dominant arm, after activation with muscle activation technique employed. These results, although with such a small sample denote the analytical techniques that improve muscle activation. Key words: EMG, FORCE, SHOULDER, MAT, NEUROPROPIOCEPTION.
\end{abstract}

Corresponding author. Department of Physichal Education, Universidad Católica de Valencia, Spain.

E-mail: esther.blasco@ucv.es

Submitted for publication January 2015

Accepted for publication March 2016

JOURNAL OF HUMAN SPORT \& EXERCISE ISSN 1988-5202

(c) Faculty of Education. University of Alicante

doi:10.14198/jhse.2016.112.06 


\section{INTRODUCTION}

The human movements, basics as those requiring more complex are performed by the contraction of skeletal muscles. All motor actions are therefore come determined by the afferent information reaching our senses way to the Musculoskeletal System ${ }^{1}$.

This mechanical information travels to the CNS where it is integrated with information from other levels, to produce motor responses that stabilize a joint or generate coordinated movement patterns in a feedback process ${ }^{2}$. The mechanical receptors that capture propioceptive information called proprioceptors ${ }^{3}$.

It is important to note the difference between information from somatic alpha and gamma motor neurons, and this difference is the technique muscle activation in this experimental study, the technique MAT $\circledast$. Firstly, the alpha motor neurons are direct responsible for the muscle to generate force, each one and together with the extrafusal muscle fibers innervating form neuromuscular union or endplate ${ }^{4}$.

Based on this muscle contraction of trapezius and relevant muscle of trunk vary, because since their contribution to the movement is essential to assist in the link of the upper extremities with the trunk. Specifically, the upper beam is in the back of the chest ${ }^{5}$, with its origin at the occipital bone, nuchal ligament by the spinous processes of all cervical vertebrae ${ }^{6}$. This muscle belongs to the acromioclavicular joint, which corresponds to the second group of the shoulder joint complex, along with the scapular-thoracic and sternoclavicular. As muscle Deltoid surrounding the shoulder joint and connects the shoulder girdle to the humeral shaft ${ }^{5}$, in this sense functions performed are different for each beam will depend on the group of fibers that act:

The clavicular portion, flexor, abductor and internal slightly rotator; The acromial abductor portion; Spinal portion: has more fibers are superior abductor, stockings or lower fibers are extensor adductor and external; All fibers are spinal extensor ${ }^{5}$. In addition, latissimus dorsi muscle is synergist of trapezium, being plane and triangular muscle, covering the lower back and the last six thoracic vertebrae ${ }^{1}$.

At present there are different techniques used to measure the activation of skeletal muscles, including the most commonly used are the electromyogram (EMG). Muscle Activation Techniques (MAT®), studied in this paper are intended to measure the proprioceptive-reflex response to a given stimulus.

The EMG is considered a reliable tool for recording muscle electrical activity, constituting an extension of the physical examination and testing of the integrity of the motor system 7,8,9. Also determined at each moment if the muscle is active or inactive 10,11 , the level of activity that has during periods in which it is active and what kind of relationship have with the rest of muscles involved in the action that is to be studied (concept of intermuscular coordination) ${ }^{12}$. Importantly, when there is a neuromuscular disease, changes are shown in the analysis of the electromyographic signal. In addition it can also be analyzed by EMGS, the phenomenon of coactivation understood as the existence of activity in agonist and antagonist muscles ${ }^{13}$.

Another technique to highlight in muscle measurements are rated with goniometry joint, being one of the most commonly used techniques for assessing joint angles. In that position goniometric examination to ensure the possibility of achieving the highest arch physiological movement ${ }^{14,15}$.

Currently there are techniques that have neuropropioceptives test responses, such as technical Muscle Activation Techniques (Muscle Activation Techniques) MAT @ , which unlike the previous two techniques has 
not been studied in depth yet, consisting of little knowledge scientist who abale in science not the case in the applied field. The main objective of these techniques is to assess the state of the neuromuscular system in order to check if the connection between the muscular and nervous systems is correct, therefore, a muscle who presents neuropropioceptive weakness (poor muscle connection - nervous system) will be unable to control the forces that reach the joints and can even cause damage to the structure (knee, spine, shoulder, tendon, ligament, capsule, etc..). The technique considers MAT ® pain ROM loss as an indicator that something is not working right and looking which is the cause that leads by assessing the neuromuscular system through tests, based on biomechanical and neurophysiological principles. Once identified muscle failure become treated in order to ensure stability and muscle control. Further, the pain may be a symptom resulting from this instability.

Conventional therapeutic treatments focus on the tense "stiff" muscles as the main factor to chronic pain or injury. The basis of MAT ${ }^{\circledR}$ is a stiff muscle is a symptom, corresponding to muscle weakness (proprioceptive). When the body senses weakness or inhibition in a muscle, joints associated become unstable, causing other muscles to increase their stress due to a lack of proprioceptive input, thereby observing a decrease in ROM. The conventional procedure is to perform a stretching tight muscles, however, if the weakness is not approached, has not approached the problem at source so it will again produce tension in the muscles to give security to the joint. In summary, instead of trying to oppression "tension" (symptom) MAT $®$ technique focuses on the cause (weakness / inhibition).

Another way to measure muscle activation is by the Borg scale, more common to measure perceived exertion ${ }^{16}$ used to provide a subjective rating of the same tool ${ }^{17}$, as it is mainly based on the component of stress ${ }^{18}$, this rating means a numerical value between zero and ten. This Borg scale is recommended by its psychometric properties ${ }^{19}$. This control method has the advantage of not requiring the use of equipment or other forms of control, however, if the specialist of Borg scale combining with other methods can get better results 20 .

The aim of this study is understands muscle activation, MAT $\circledast$ technique, contrasted with other known techniques with a scientific prestige in the assessment of trapeze superior beam and observe their influence on the behavior of the three bundles of the deltoid (like muscles agonists) and the latissimus dorsi muscle (antagonist muscle).

\section{METHOD}

\section{Participants}

The sample consisted of 8 women $(35.3 \pm 1.3$ years), which signed an informed consent to conduct the study. Of the eight participants who underwent the test of MAT technique, five of them gave a positive result and three of them negative, so were rejected for carrying out the work. They were informed and accepted their voluntary participation by signing a consent report, under the ethical guidelines of the Declaration of Helsinki of the World Medical Association (updated in the World Medical Assembly in Seoul 2008), for research involving humans. For inclusion in the study, subjects had to meet the following criteria: being a woman, hitting positive answer in test Neuropropioceptive test, not present injury or shoulder pain.

\section{Procedure and Protocol}

First we proceeded to perform the check of upper trapezius of both shoulders by the Muscular Activation specialist, in order to verify proper or improper functioning and assess whether it is positive or negative. For this assessment, we realized the neuropropioceptive response test, which consisted of an assessment of 
neuromuscular weakness, for which the subject is placed on a stretcher in supine, with the arm in a standardized abduction of $120^{\circ}$ and internal rotation also, makes held shoulder position where shortening of the fibers of the upper trapezoid is achieved, and also pull the other plane force that can intervene abductor muscles during the test, namely, in order to avoid possible offsets. Once the subject is positioned and is applied to an approximate force of $10 \mathrm{Kg}$, constant and consistent, in a direction toward approaching the arm adducted hip. This is the moment when the subject has to resist the force preventing movement of the arm for at least two seconds. If the professional forces move the body part of the participant, the result would be positive associating an ineffective and weak upper trapezius neuropropioceptive response, unlike when the individual itself is capable of withstanding where this answer can be understood as effective and the test would negative. The variable approximate $10 \mathrm{Kg}$ of force applied and the time during which we must resist, is determined and provided by the U.S. creator (Roskopf) of Muscle Activation Techniques ${ }^{\circledR}$.

Once any of the trapezoids had tested positive for the palpation, the sample is valid for study and intervention following protocol is followed:

Initially, we realize a heating for the subject of 2 minutes duration, which is used theraband and weighs 10 $\mathrm{kg}$ is performed. By giving positive, we will proceed to study the subject performing goniometric both shoulders to thereby objectify joint range. Measurements are made as Flexion and Abduction.

Once finally goniometric measurement, we proceeded to check both trapezoids using the appropriate MAT ( $)$ test technique that has been used previously. Should be positive neuromuscular weakness will proceed to complete the test according to the Borg scale of perceived exertion made between parameters from 0 to 10 21. After the palpation, the measurement was performed with surface electromyography, this will shaved the area where the electrodes were placed and proceeded to clean with alcohol and cotton.

Then the position of the electrodes depending on the muscle is described:

1. Upper Trapezius: two active electrodes $2 \mathrm{~cm}$ apart parallel to the muscle fibers (origins and insertions) of the upper trapezius, along the crest of the shoulder, slightly lateral and midway between the cervical spine $\mathrm{C}-7$ and the acromion. Should be palpated muscle mass and place the electrodes on the muscle belly ${ }^{22}$, a third neutral electrode was mass effect ${ }^{23}$.

2. Anterior Deltoid: for this we must feel the collarbone and two active electrodes placed $2 \mathrm{~cm}$ apart from each other in the front of the arm, $4 \mathrm{~cm}$ below the clavicle above and parallel to the muscle fibers ${ }^{22}$, a third electrode made neutral mass effect 23 .

3. Medium Deltoid: The active electrodes are placed on the top side of the arm, $2 \mathrm{~cm}$ away from each other and $3 \mathrm{~cm}$ from the acromion, on muscle mass so that the electrodes run parallel to the muscle fibers ${ }^{22}$, a third neutral electrode was mass effect ${ }^{23}$.

4. Posterior Deltoid: You have to feel the spine of the scapula, two electrodes at $2 \mathrm{~cm}$ distance between them and $2 \mathrm{~cm}$ below the side of the spine of the scapula and are placed at an oblique angle to this arm so that run parallel to the muscle fibers ${ }^{22}$, a third neutral electrode was mass effect ${ }^{23}$.

5. Latissimus Dorsi: Both electrodes $2 \mathrm{~cm}$ distance between them and at T12 line between armpit and looking for the edge of the scapula so they run parallel to the muscle fibers 22 are placed third neutral electrode was mass effect 23 . 
Once the electrodes located distributed among the following 10 channels in both arms (Right Zone and Left Zone).

Previous to electromyographic recording, an isometric test was performed using a maximal contraction (10 seconds), in order to use it to normalize the data collected, namely, a maximum isometric contraction and EMG activity was recorded on MRI. Then the electromyographic measurement was performed by realizing the same movement as that which initially found that the upper trapezius is inhibited by the MAT $®$ technique.

Finally, the MAT ${ }^{\circledR}$ technique is performed using a very precise muscular pressure, in order to restore communication between the muscle and the nervous system. Once completed it, we realize again the neuropropioceptive test in the same position where he had failed a principle to verify the result after activation. It then proceeds to collect records Borg Scale after the last test in the same way as before using the scale of 0 to 10 . Turn again recorded with electromyography after performing muscle activation and finally we held back the bearing measurement if there is any variation in the initial records.

\section{Data analysis}

The statistical treatment of the results was performed using the statistical software (SPSS) 22.0 for Windows. First the normality test of Kolmogorov-Smirnov, showing the nonparametric distribution of the data was performed, while for the data analysis, we used the T-samples to compare the pre and post test activation. The value of $p<.05$ was used to determine statistical significance.

\section{RESULTS}

First descriptive data of the sample in the studied variables, as can be seen in Table 1 are shown:

Table 1. Electromyographic Characteristics, ROM and Borg Scale

\begin{tabular}{lcc}
\hline & Mean & Maxim \\
Right Trapezius Pre & $116,4 \pm 76,2$ & $201,8 \pm 76,2$ \\
Left Trapezius Pre & $164 \pm 102,3$ & $279 \pm 153,4$ \\
Right Trapezius Post & $122,6 \pm 72,9$ & $217,2 \pm 130,8$ \\
Left Trapecio Post & $139,4 \pm 88,8$ & $230 \pm 154$ \\
Right Clavicular Pre & $238,8 \pm 94,3$ & $844 \pm 779,5$ \\
Left Clavicular Pre & $157 \pm 96,4$ & $281,4 \pm 187,4$ \\
Right Clavicular Post & $250,4 \pm 147,1$ & $472,6 \pm 214$ \\
Left Clavicular Post & $147,4 \pm 104$ & $241 \pm 142,2$
\end{tabular}




\begin{tabular}{|c|c|c|}
\hline Right Acromial Pre & $221,2 \pm 100,2$ & $469,2 \pm 151,3$ \\
\hline Left Acromial Pre & $279,4 \pm 267,1$ & $470,4 \pm 466,3$ \\
\hline Right Acromial Post & $189,6 \pm 118,7$ & $391,8 \pm 136,1$ \\
\hline Left Acromial Post & $262 \pm 270,7$ & $427,2 \pm 406,5$ \\
\hline Right Spinal Pre & $141 \pm 92,9$ & $259,8 \pm 176,4$ \\
\hline Left Spinal Pre & $68,6 \pm 40,1$ & $107,4 \pm 57,9$ \\
\hline Right Spinal Post & $107 \pm 79,2$ & $197,2 \pm 156,4$ \\
\hline Left Spinal Post & $61,2 \pm 21,5$ & $107,6 \pm 59,4$ \\
\hline Right Latissimus Dorsi Pre & $22,4 \pm 92,9$ & $259,8 \pm 176,4$ \\
\hline Left Latissimus Dorsi Pre & $27 \pm 20,3$ & $52,2 \pm 37,2$ \\
\hline Right Latissimus Dorsi Post & $13,4 \pm 12$ & $36,6 \pm 30,4$ \\
\hline Left Latissimus Dorsi Post & $16,4 \pm 10,9$ & $29,2 \pm 19,5$ \\
\hline Right Flexion Pre & $173,6 \pm 4,7$ & ----------- \\
\hline Left Flexion Pre & $175 \pm 3,7$ & 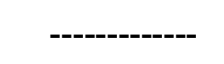 \\
\hline Right Flexion. Post & $176,2 \pm 2,5$ & --------- \\
\hline Left Flexion Post & $177 \pm 2$ & ---------- \\
\hline Right Abduction Pre & $173,6 \pm 4,1$ & ------ \\
\hline Left Abduction Pre & $174,2 \pm 3,8$ & ----- \\
\hline Right Abduction Post & $176,2 \pm 2,2$ & ----- \\
\hline Left Abduction Post & $177 \pm 1,8$ & ----- \\
\hline Right Borg Scale Pre & $7,2 \pm 2,4$ & ----- \\
\hline Left Borg Scale Pre & $8,2 \pm 1,8$ & ------------ \\
\hline Right Borg Scale Post & $4,2 \pm 3$ & \\
\hline
\end{tabular}


The results between the pre and post muscle activation of the upper trapezius beam, show significant differences (Table 2$)$ between the mean values $(p=.021)$ and maximum $(p=.001)$ of the right trapezius, also at the left trapezius, in the mean $(p=.002)$ and the maximum values $(p=0.012)$. It has also noted a curious fact regarding the activation of the left trapezius has shown significant improvements in the right shoulder in both the mean $(p=.008)$ and high $(p=.024)$.

In relation to the electomyografic study of deltoid, it will be divided into the three beams studied by the clavicular result shows a significant activation in the right middle $(p=.012)$ and maximum left $(p=.003)$, as showing in the trapezoid that activation of the left arm affects significantly the activation of the right average $(p=.005)$. In the spinal tract results obtained are scarce, denoting a significant improvement in the average $(p=.003)$ and the maximum right $(p=.024)$. Where significant differences were found in all analyzed electromyographic variables in the acromial beam is both the right middle $(p=.01)$ and high $(p=.038)$ and in the left middle $(p=.003)$ and high $(p=.002)$, along with the influential maximum activation in the left middle right side $(p=.000)$ and $(p=.038)$ (Table 2$)$.

Table 2. Relation Pre and Post activation value with MAT technique.

\begin{tabular}{lccccc}
\hline & Trapezius & Clavicular & Acromial & Spinal & Latissimus Dorsi \\
\hline R.R. Mean & .021 & .012 & .01 & .003 & .017 \\
R.R. Maxim & .001 & --- & .038 & .024 & .068 \\
L.L. Mean & .002 & --- & .003 & --- & .02 \\
L.L. Maxim & .012 & .003 & .002 & --- & .038 \\
L.R. Mean & .008 & .005 & .000 & --- & .003 \\
L.R. Maxim & .024 & --- & .000 & --- & .005 \\
R.L. Mean & --- & --- & --- & --- & .000 \\
R.L. Maxim & --- & --- & --- & --- & .000 \\
\hline
\end{tabular}

Legend: $R=$ Right, $L=$ Left

Turn the latissimus dorsi displayed on all variables measured by electromyograph significant, such as in the right half $(p=.017)$, not being in a high but shows a marginal trend $(p=.068)$ values, as average $(p=.02)$ and a maximum left ( $p=.038)$. addition to activation in the left and its influence on his right shoulder in the middle $(p=.003)$ and high $(p=.005)$. It should be noted that it is the only muscle that result also shows significant activation in the right and its influence on the left in both the mean $(p=.000)$ and high $(p=.000)$. Besides all the above findings was noted that the results for the measurement with the test response 
neuropropioceptivos were highly significant $(p=.000)$, which makes us think about the need for further study of this novel technique and its impact in sports performance.

\section{DISCUSSION}

Although this study could say it's the beginning or starting point for the following work related to Muscle Activation Techniques MAT $®$, the results obtained with such a small sample as evidenced by the significant differences found in most muscles tested.

Thus based on the biomechanics of the anatomic situation premise of these muscles, the results obtained show the offsets and synchronicity between them ${ }^{5}$.

When analyzed individually each examined muscle, upper trapezius beam shows significant improvements in all variables studied, on the basis that the muscle activation technique used was specific to this muscle, the results presented consistent with the objective of this work. Since this muscle plays a role of scapular stabilization acting basis for movements of upper extremities, presenting a driving role in the shoulder girdle, by the rhomboids, elevator scapulae, serratus anterior, pectoralis minor and subclavian 5 is consistent to think that the technique has improved functionality to use a range of travel according to their anatomical arrangement.

Therefore is possible that this improvement is due to the activation and technique, although further studies would be needed in this line in order to draw a conclusion consistent with this result.

Regarding the results obtained in the rest of the muscles analyzed, controversy exists between the results obtained, showing significant evidence especially in synergist muscle Deltoid Acromial Portion in all variables analyzed and the latissimus dorsi muscle antagonist. These results may show muscle joint and muscle substitutions found in sports and everyday activity in all movements executed, working on muscle chains work ${ }^{10}$.

In this regard, has noted that contralateral very high correlation between the upper trapezius muscle bundle and the dorsal, which suggests that the latter had greater muscle activation to curb traction from the opposite shoulder is being made to be seen adduction and thus challenging the contralateral lateral flexion, this muscle should be avoided, something that happens when the test is positive due to the loss of strength of shoulder abductors themselves ${ }^{5}$.

In this sense, this work shows a muscular activation using a novel technique to date, which is recorded with electromyographic a significant improvement in all muscles analyzed, so it is necessary to continue analyzing this line to contrast these results and strengthen the usefulness of these techniques.

\section{REFERENCES}

1. Sobotta, J. (2000), Atlas of Human Anatomy. Elsevier Science Publishing. New York

2. Myers, J., \& Lephart, S. (2000.) The Role of the Sensorymotor System in the Athletic Shoulder. $J$ Athl Train 35(3), 351-363.

3. Myers, J., \& Oyama, S. (2008). Sensorimotor factors affecting outcome following shoulder injury. Clin Sports Med 27, 481-490.

4. Stevens, A., \& Lowe, J. (1999). Histologie Humaine. Elsevier Science Publishing: New York. 
5. Kapandji, A. I. (2006). The Physiology of the joint. Volume Three (trunk and Vertebral Colum). Churchill Livingstone.

6. Schünke, M., Schulte, E., Schumacher, U., \& Wesker, K. (2006). Prometheus: Panamericana.

7. Basmajian, J.V. \& De Luca C.J. (1985). Muscles Alive. Their functions revealed by electromyography. Baltimore: Williams \& Wilkins.

8. Cram, J.R., Kasman, G.S., \& Holtz, J. (1998). Introduction to surface electromyography. Gaithersburg: Aspen publishers.

9. Kimura, J. (1983). Electrodiagnosis in diseases of nerve and muscle. Philadelphia: FA Davis.

10. Delagi, E. \& Perotto, A. (1981). Anatomic guide for the electromyographer. Springfield, IL: Charles C. Thomas.

11. Balestra, G., Frassinelli, S., Knaflitz, M., \& Molinari, F. (2001). Time- Frequency analysis of surface myoelectric signals during athletic movement. IEEE Engineering in Medicine and Biology, 20(6), 106115.

12. Potvin, J.R., \& Bent, L.R. (1997). A validation of techniques using surface EMG signals from dynamic contractions to quantify muscle fatigue during repetitive tasks. Journal of Electromyography and Kinesiology, 7(2), 131-139.

13. Cramer, J.T., Housh, T.J., Weir, J.P., Johnson, G.O., Ebersole, K.T., Perry, S.R., \& Bull. A.J. (2002). Power output, mechanomyographic, and electromyographic responses to maximal, concentric, isokinetic muscle actions in men and women. J. Strength Cond Res, 16(3), 399-408.

14. Rowe, C. R. (1974) Adult re-evaluation of the position of the arm in arthrodesis of the shoulder in the adult. J Bone Joint Surg Am, 56-A, 913-922.

15. Norkine \& White (2006). Measurement of Joint Motion: A Guide of Goniometry. F.A. Davis Company. Marbán: Spain.

16. Coquart, J. B., Tourny Chollet, C., Lemaitre, F., Lemaire, C., Grosbois, J. M., \& Garcin, M. (2012). Relevance of the measure of perceived exertion for the rehabilitation of obeses patients. Annals of physical and rehabilitation medicine, 55(9-10), 623-640.

17. Matoulek, M. (2007). Defining the level of physical activity for a diabetic who is obese. Vnitrni Lékarství, 53(5), 560-562.

18. Mäestu, J., \& Jürimae, T. (2005). Monitoring performance and training and rowing. Sports Medicine, 35(7), 597-617.

19. Gerlach, Y., Williams, M. T., \& Coates, A. M. (2012). Weighing up the evidence a systematic review of measures used for the sensation of breathlessness in obesity. International Journal of obesity, 37(3), 341-349.

20. Borg, E. \& Kaijser, L. (2006). A comparison between three rating scales for perceived exertion and two different work tests. Scandinavian Journal of Medicine \& Science in Sports, 16(1), 57-69.

21. Borg, G. (1982). Psychophysical bases of perceived. J.Med.Sci.Sports Exercise, 14(5), 377-381.

22. Cram, J.R. (2005). Cram's introduction to Surface Electromyography. Jones and Bartlett Publishers: Sudbury, Massachusetts.

23. Clarys, J.P. \& Cabri, J. (1993). Electromyography and the study of sports movements: a review. J. Sports Sci, 11(5), 379-448. 